\title{
Comparison of Different Spatial Interpolation Techniques of Field Measurements to Construct Radio Environment Map
}

\author{
K. Bhaskar Reddy ${ }^{1 *}$ and P. Venkatramana ${ }^{2}$ \\ ${ }^{1} P G$ Scholar/ECE, Sree Vidyanikethan Engineering College, Chittoor, AP \\ ${ }^{2}$ Professor/ECE, Sree Vidyanikethan Engineering College, Chittoor, AP \\ 1*kr182454@gmail.com, ${ }^{2}$ ramanasree_pv@yahoo.co.in
}

\begin{abstract}
Still, Spectrum has become one of the crucial needs of living in the modern world. Wireless Communication has increased wide prominence in the current years. From cellular voice telephony to wireless access to the internet and wireless home networking, wireless networks have a profound impact on our lifestyle. However, the emerging needs in wireless communications are making the spectrum congested, and hence there might be a shortage of spectrum in the future. Cognitive Radio $(C R)$ is a niche technology to extend the effective utilization of spectrum by the opportunistic usage of idle spectrum resource smartly. To construct REM there are many techniques which can be categorized as direct and indirect methods. Direct methods involve spatial interpolation of the available data on measured locations to estimate the values at unmeasured locations. To estimate different spatial interpolation techniques, the information utilized in this paper is from the WiseProject Measurement Report: "PMSE Protection Measurements in Helsinki City Theatre" and applied interpolation techniques in ArcGIS software such as Inverse Distance Weighting (IDW), Voronoi interpolation, Ordinary, Universal and Indicator Kriging. Precision in estimation is measured using cross validation of root mean square error [RMSE], and it is utilised for the comparative analysis of interpolation methods applied to the field measurement information.
\end{abstract}

Keywords: Cognitive Radio (CR), Radio Environment Map (REM), spectrum sensing, Spatial Interpolation, IDW, Spline interpolation, Radial basis function, Nearest Neighbourhood, Kriging, ARCGIS etc

\section{Introduction}

Recently, with the step increase in the number and type of wireless devices, the unlicensed spectrum becomes increasingly scarce. Radio Frequency (RF) is an infrequent resource, and hence its proper utilization is very important, now more than ever. Discovering the spectrum band to meet demands of future wireless services is becoming more and more difficult. CR is a favorable technique for efficient exploitation of spectrum by introducing cognitive and reconfigurable ability to the conventional radio system. For more about CR refer [1].

The idea for CR was obtained by Joseph Mitola at the Defense Advanced Research Projects Agency (DARPA) in the United States. It is a smart radio, which is conscious of its surrounding environments and proficient of changing its behaviour to optimize the consumer experience $[1,2]$. Cognitive radio (CR) is a method of wireless communication in which a transceiver can cleverly detect which communication channels are in use and which are not, and quickly move into empty channels while getting away from involved ones. It can learn from the radio surrounding conditions and can change to fit the changes

Received (January 4, 2018), Review Result (March 26, 2018), Accepted (March 29, 2018)

* Corresponding Author 
in it. Today's wireless networks are worked by the basic rule of static spectrum assignment policy [2]. Hence, one core feature of cognitive radio is interrelated to autonomously exploiting local unused spectrum to provide new tracks to spectrum access. According to FCC, not every channel in every band is always in use [3]. CR operation involves gaining knowledge, making decisions and reconfiguring radio parameters [5]. The motive for Cognitive radio is a perception of utilizing licensed spectrum in an unlicensed manner without affecting interference [4].

To execute without Obstruction to the primary signal, the cognitive radio wants to sense the usage of the spectrum before getting to the channel [4].

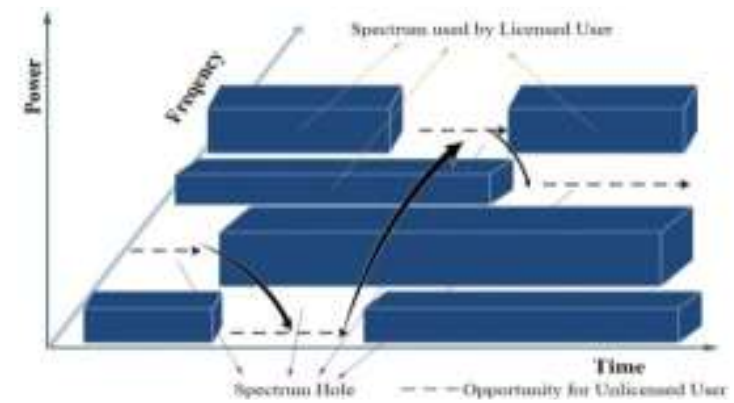

Figure 1. Representation of White Spaces

So the capability of identifying an idle spectrum and the ability to briefly access a spectrum without intrusive with Primary Users are two extremely important components required for the accomplishment of cognitive radios [5]. In cognitive radio terminology, Primary Users (PU) can be characterized as the user who has the permit to use a particular part of the spectrum. On the other hand, secondary users (SU) do not have permit to use the spectrum but only when PU is inattentive. Cognitive radio can detect the unfilled frequency channels for the secondary users when primary user is not devouring the allotted frequency spectrum, so that spectrum usage can be improved. Cognitive radios are that it ought not to meddle with the authorized clients and ought to abandon the band when required. For this, it must detect the signals quicker [6]. For more information on spectrum sensing, referred the [7-10].

\section{Radio Environment Maps [REM]}

REM was presented by the Virginia Tech group. Much the same as a city delineate guides the voyager, REM empowers the working of CR [11]. It is a concentrated or dispersed database containing data on the radio surrounding conditions including:

- Device locations and their activities,

- Policies and regulations,

- Geographical features i.e., status of spectrum utilisation, coverage regions, and interference levels

- vacant radio systems and their allotted frequency bands,

REM planned for IEEE 802.22 to develop TV White Spaces (TV WS). Our idea of REM is more general than the available database approach described above [11]. We depict REM as a keen substance which stores incoming environmental data but also interpolates this data to profit from the spatial correlation that exists in the data. The thought of gathering geo-localized data on the radio environment and creating a map using this information has also been inspected and developed further by other research groups [6]. In this mechanism, REM has held in a more general Cognitive Radio (CR) 
context than TV white spaces and it is considered as a mean to represent Spatiotemporal attributes of the radio environment by using concepts and tools from spatial statistics [12], like point processes, spatial random fields, pair correlation functions, point interaction models, spatial interpolation techniques, etc.

Cognitive radio uses the spectrum sensing techniques [7-10] and requires spectrum awareness, which gives information on spectral occupancy of an under-utilized wireless system. Such information can be handover in the form of a map called as Radio Environment Map (REM) [11]. REM represents the occupancy of a channel at any given location using either field measurement or database.

REM Construction: The accumulated information of REM is predicated on the terminals measurement data, cumulates with location data and reported to a functional entity. These entity feats this information to build a consummate map by interpolating the geo-localized measurements. Because quantification reporting is sumptuous in terms of signaling overhead and battery consumption, the main contest while building the REM is to find the optimal trade-off between the REM quality, i.e., the REM data accurateness and the measurements requested from the terminals [12].

\section{Spatial Interpolation Techniques for REM}

Spatial interpolation techniques are direct methods for building REM, such spatial continuous surfaces of radio fields can be generated by applying interpolation techniques to the sampled field measurements [14]. Such methods consider the spatial relationship between the sampled data points and define a function for estimation at other locations.

In general, it is hard to measure the parameters at each and every location of the area under test. Spatial interpolation is the process of using a set of point data to engender surface data. A point data set has data values only for certain locations, such as fieldwork locations, within the study zone. Surface data divides the study zone into cells, with a data value for each cell. With surface data, there is often a data value for every location inside the study zone, whether it was sampled or not. Though a set of point information is more manageable in terms of labor, budget, and time; surface data are more useful and practical in many disciplines, such as precision agriculture [13], particularly with variable rate applications. There are many spatial interpolation algorithms available in the literature, as well as in commercial GIS or statistics software [16]. Each algorithm usually requires different parameters. Even with the same algorithm and same input data points, these different parameters can create dissimilar surfaces.

Estimation of interpolated surfaces is difficult and often unnoticed. In maximum spatial interpolation research, quantitative assessment becomes the best technique used to estimate the resultant surfaces. Most quantitative strategies offer a numeric index for usual performance. This type of numeric index is simple to understand and convenient [14-16]. However, interpolated surfaces cannot be described by one numeric index, as many characteristics cannot be observed or evaluated by quantitative assessments.

There are two rudimental prospects for spatial interpolation. First is spatial autocorrelation, which is best described by Tobler's first law of geography "everything is cognate to everything else, but near things are more cognate than distant things" [15]. The second postulation is that values are smooth and perpetual over space. Many spatial interpolation techniques were developed predicated on these two posits. Commercial GIS or statistical software provides several spatial interpolation techniques, such as inverse distance weighted (IDW), kriging, Spline, and others [15].

Although there are many opportunities for spatial interpolation, to date, there is no "rule of thumb" on which technique is best under what certain circumstances. Even with the same technique and same input point data, different parameters may outcome in different surfaces. Potentially, a specified set of points and a given spatial interpolation technique can create many different surfaces $[14,15]$. Consequently, it is vital to calculate and 
understand the accuracy and reliability of floor information generated from spatial interpolation. In this study, IDW, kriging, and Spline will be habituated to establish the process to evaluate and visualize spatial interpolation surfaces.

Spatial interpolation techniques can be classified as global and local interpolation, exact and inexact interpolation, and deterministic and stochastic interpolation [16]. Global interpolation is the method which uses whole sampled values for prediction, whereas local interpolation uses the sampled values at their local range. The Classification of Spatial Interpolation Techniques as shown in Figure 2.

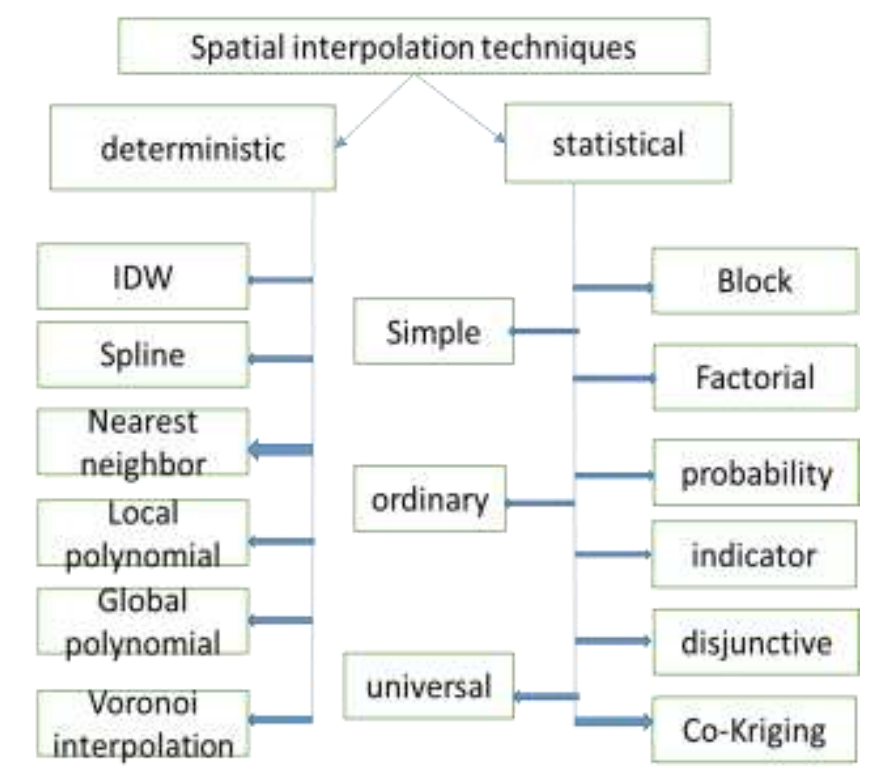

Figure 2. Classification of Spatial Interpolation Techniques

Interpolation techniques whose predicted and measured values at all sample locations are the same, are the exact interpolation; otherwise, it will be an inexact interpolation. Deterministic interpolation techniques produce the continuous surface using either the degree of smoothing or the similarity extent [15]; whereas statistical interpolation deals with the spatial autocorrelation of the quantified data i.e., it utilizes the statistical enquiry of the data to engender the surface [16].

The quality of sample point set can perturb cull of interpolation method as well. If the sample factors are poorly disbursed or there are few of them, the surface may not constitute the real terrain very well. The real-world awareness of the difficulty rely will first of all affect which interpolation technique to apply.

\section{A. Deterministic Interpolation Techniques}

\section{1). Inverse Distance Weighting (IDW):}

Inverse distance weighted is a pre-defined guess method where values at unmeasured points are figured out by a linear combination of values at adjacent measured points. Among available parameters, the power parameter can appreciably affect the outcomes. As the power parameter rises, IDW performances in addition to the nearest neighbor interpolation method in which the interpolated value is proximate to the value of the most proximate quantified value. The benefits of IDW are that it is simple, facile to understand, and efficient. Drawbacks are that its miles touchy to outliers and there is no hint of error [17]. The weight factor depends on the distance between the measured $(\mathrm{x})$ and predicted locations (xi) i.e., $\mathrm{d}(\mathrm{x}$; $\mathrm{xi})$. The weight factor $\mathrm{w}_{\mathrm{i}}(\mathrm{x})$ is given by: 


$$
\mathrm{w}_{\mathrm{i}}(\mathrm{x})=\frac{1}{\mathrm{~d}\left(\mathrm{x}, \mathrm{x}_{\mathrm{i}}\right)^{\mathrm{p}}}
$$

\section{2). Nearest Neighbour Interpolation:}

The Nearest Neighbour technique is the naivest spatial interpolation from a computational point of view, where the value consigned at each output location is the value of the nearest sample data point to that location. However, it is a modest technique because it fails to consider the influence of the sample data points apart from the nearest neighbouring data point. In this method, only the closest neighbours will be considered for calculations [16]. This method is poorer in complexity as well as accuracy. It is also known as proximal interpolation, or point sampling [17].

\section{3). Spline Interpolation:}

Spline interpolation, also well-known as "radial basis function", is one of the exact interpolation methods i.e., the surface created by prediction will continuously pass through the measured values [16, 17]. A most important advantage of Spline is that it can generate properly accurate and visually attractive surfaces based on only limited sample points.

Drawbacks of Spline are that the consequent surface might have different minimum and maximum values from the input data set, it is sensitive to outliers, and there is no indication of errors. It is the technique of minimizing the total curvature by fitting the flexible membrane. Spline interpolation produces interpolated values even beyond the most and minimal measured values, whereas in IDW it will be under the limits of measured values.

\section{4). Thiessen or Voronoi Interpolation:}

In this method, set of Voronoi polygons are created surrounding the measured sample points, as shown in Figure 3. Creation of Voronoi polygons contains Delaunay triangles whose sides are designed by connecting sample points.

If we draw a circle passing through three corners of Delaunay triangles, then that circle will not enclose any sample points inside. This is the essential property of Delaunay triangles. If a sample point exists, then we need to rearrange the corners of the triangle [17]. There are numerous ways to assign values to each polygon, and they are defined elaborately in reference [14].

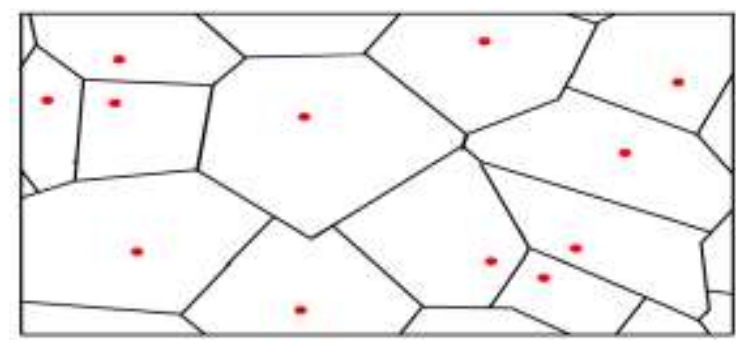

Figure 3. Voronoi Cells or Theissen Polygons using Delaunay Triangles

\section{5). Local and Global Polynomial Interpolation:}

\section{i). Local Polynomial Interpolation:}

In local polynomial interpolation, the estimated value $\hat{z}\left(\mathrm{~s}_{0}\right)$ at location $\mathrm{s}_{0}$ can be calculated by fitting a linear curvature using polynomials of any order within the search neighbourhood [18]. For example we can fit a first-order polynomial as given in below equation for the given data set inside search neighbourhood.

$$
\hat{z}\left(s_{0}\right)=\widehat{\alpha_{0}}+\widehat{\alpha_{1}} x+\widehat{\alpha_{2}} y
$$


Where the parameters of the polynomials are defined by $\widehat{\alpha_{0}}$, which can be created by minimizing the sum of squared deviations and $(\mathrm{x}, \mathrm{y})$ is the coordinates of interpolating location $\mathbf{s}_{0}$.

\section{ii). Global Polynomial Interpolation:}

Global polynomial interpolation takes in to account the complete data set to fit the polynomial, rather than just local neighborhood, as in case of local polynomial. The subsequent surface is very smooth, as that it represents a gradual trend in the surface. It can also be termed a trend surface analysis. A second order trend surface can be given by:

$$
\hat{z}\left(s_{0}\right)=\widehat{\alpha_{0}}+\widehat{\alpha_{1}} x+\widehat{\alpha_{2}} y+\widehat{\alpha_{3}} x^{2}+\widehat{\alpha_{4}} x y+\widehat{\alpha_{3}} y^{2}
$$

Where $\in$ symbolizes the local variations or noise. Higher order polynomials contain a greater number of cross products along with higher powers of coordinate variables $(\mathrm{x}, \mathrm{y})$.

\section{B. Statistical Interpolation Techniques}

Kriging interpolation: Kriging is a geostatistical gridding method that has proven valuable and popular in many fields. Kriging is a stochastic technique interrelated to IDW in that it additionally uses a linear combination of weights at recognized locations to estimate the data value of an unknown region. Variogram is a significant input in kriging interpolation [14]. It is a measure of spatial correlation between two points. With wellknown variograms, weights can transform according to the spatial array of the samples. A maximum critical benefit of kriging is that further to the estimated floor, kriging also affords a measure of mistakes or uncertainty of the expected floor. A drawback is that it needs considerably more computing time and more input from users, compared to IDW and Spline. The recognized variograms can significantly raise kriging performance, which may outcome in a superior performance than IDW [20].

A powerful statistical interpolation technique used for various packages inclusive of health sciences, geo chemistry, and pollution modeling [18-20].

Simple kriging, the trend component is assumed to be known and constant.

Ordinary kriging, the trend component is assumed to be constant but unknown. It is the default kriging method as it doesn't want the expertise of the trend, and it is most practically applicable compared to other kriging methods.

Universal kriging does anticipate that an overruling trend exists in the data and that it can be modeled.

Co-Kriging: It is a multivariate kriging, where interpolation is performed using one or more correlated variables.

Indicator Kriging: It is a non-linear method of interpolation where binary data is created by using the threshold value and continuous sample data. If the sampled data value crosses the threshold, then ' 1 ' will be allotted to indicator variable, otherwise zero.

Disjunctive Kriging: It is a non-linear interpolation, where we can get the estimation of the conditional probability of an indicator variable when it crosses certain tolerance level.

Block Kriging: In this approach of kriging, data values will be interpolated to the area bigger than the actual area. It is an extension of ordinary kriging and estimates a Block value instead of a point value.

\section{Results \&Analysis}

A Here taking the field measurements from Wise-Project Measurement Report: "PMSE Protection Measurements in Helsinki City Theatre" funded by Nokia, Fair spectrum, Ficora and Wise-project on 9th of December 2011 published in the $12^{\text {th }}$ meeting of SE43 Cambridge, 13 - 16 December 2011. 
There are many methods of interpolation. Choosing an interpolation method is influenced by the awareness of the surface for modeling. Every approach running in a unique way, however, maximum makes use of the concept of spatial autocorrelation; Near matters are greater alike than things a long way away. In preference to expect one interpolation technique is better to try distinct interpolation techniques and evaluate the results to determine the greatest interpolation technique for a given project.

The field measurements can simulate in ArcGIS software [ARC Map] [16] and observe graphical and statistical parameters as followed below, Graphical wizards of field values and their signal strengths.

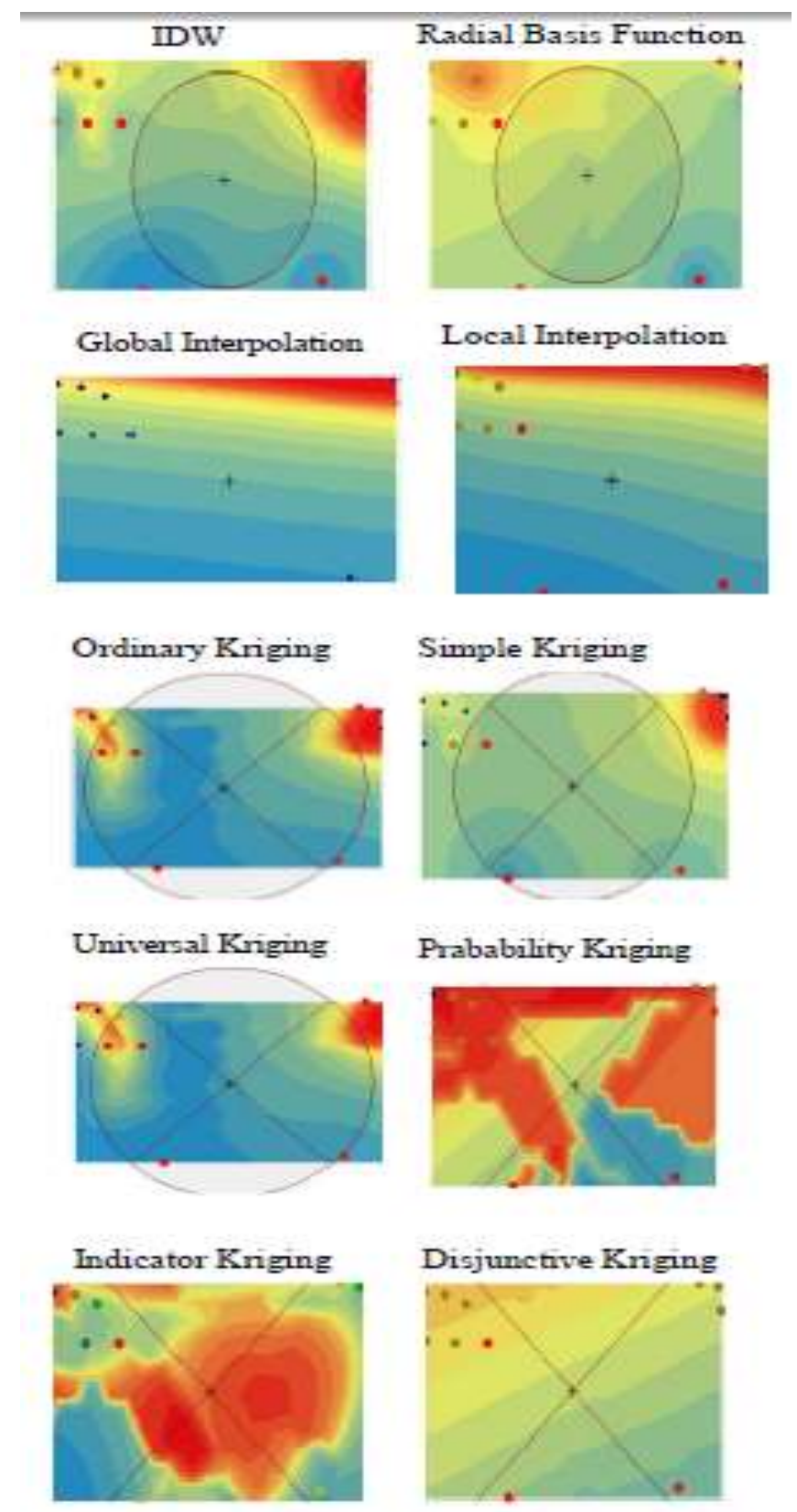

Figure 4. Geo Statistical Wizards for Interpolation Techniques

Appearing smoother with less far values is not fundamentally an indication of good performance or bad performance. It is just a quality of the overall trend of the 
interpolated surface, which was not exposed by quantitative assessment, such as error measures shown in Table 1. In the quantitative assessment, it was identified that IDW and kriging performed equally, and both are better than spline. With early visual scrutiny, it was detected that IDW and spline performed equally, while kriging performed differently, not certainly in a superior or poorer manner. Kriging achieves statistical study of the error in its predictions. This allows it to create four kinds of surfaces: prediction, standard error, quantile, and probability. Prediction maps estimate values at locations where measurements have not been taken. (All interpolators make prediction maps.)

The prediction curves are as shown below.
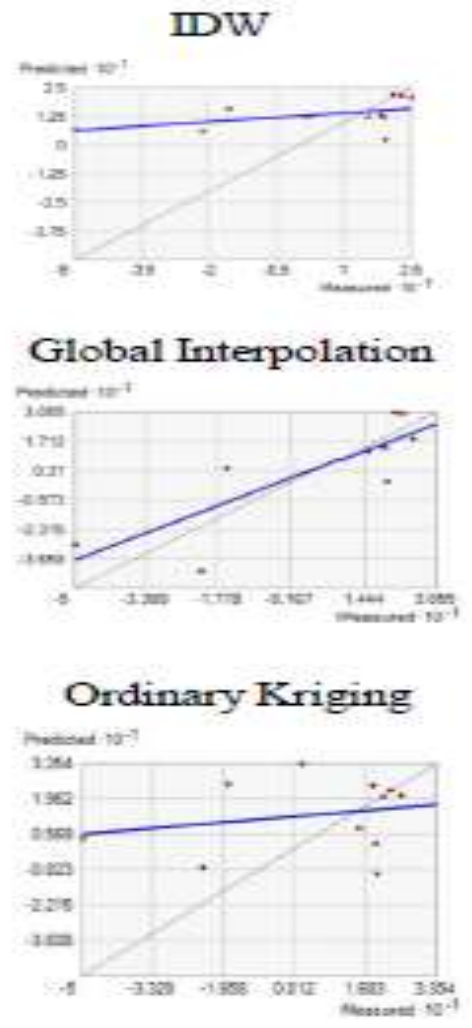

Universal Kriging

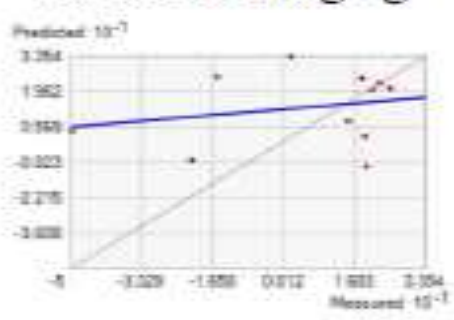

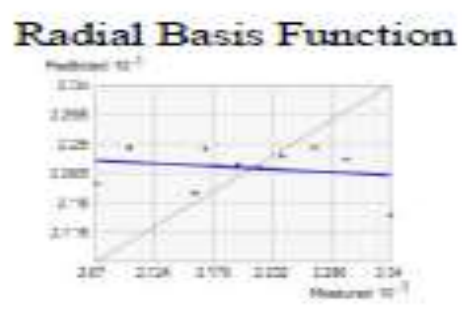

Local lnterpolation

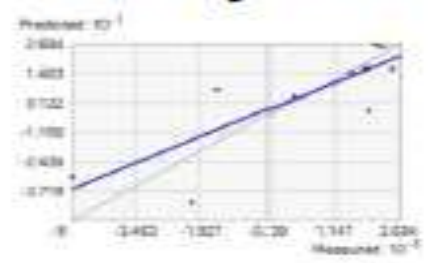

\section{Simple Kriging}

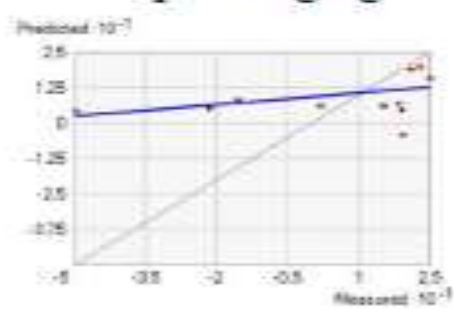

Indicator Kriging

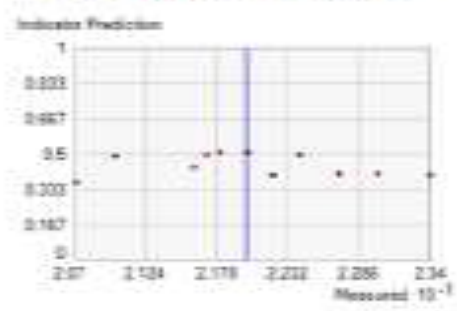

Figure 5. Prediction Curves of Interpolation Techniques

The Semivariogram takes the spatial dependency among samples by plotting semivariance against parting distance. Semivariance equivalents one-half the squared difference between points divided by a distance $\mathrm{d} \pm \Delta \mathrm{d}$ (assuming no direction preference).

Standard error maps show the distribution of prediction error for a floor. Errors have a tendency to be highest in locations where there are very little sample records. 

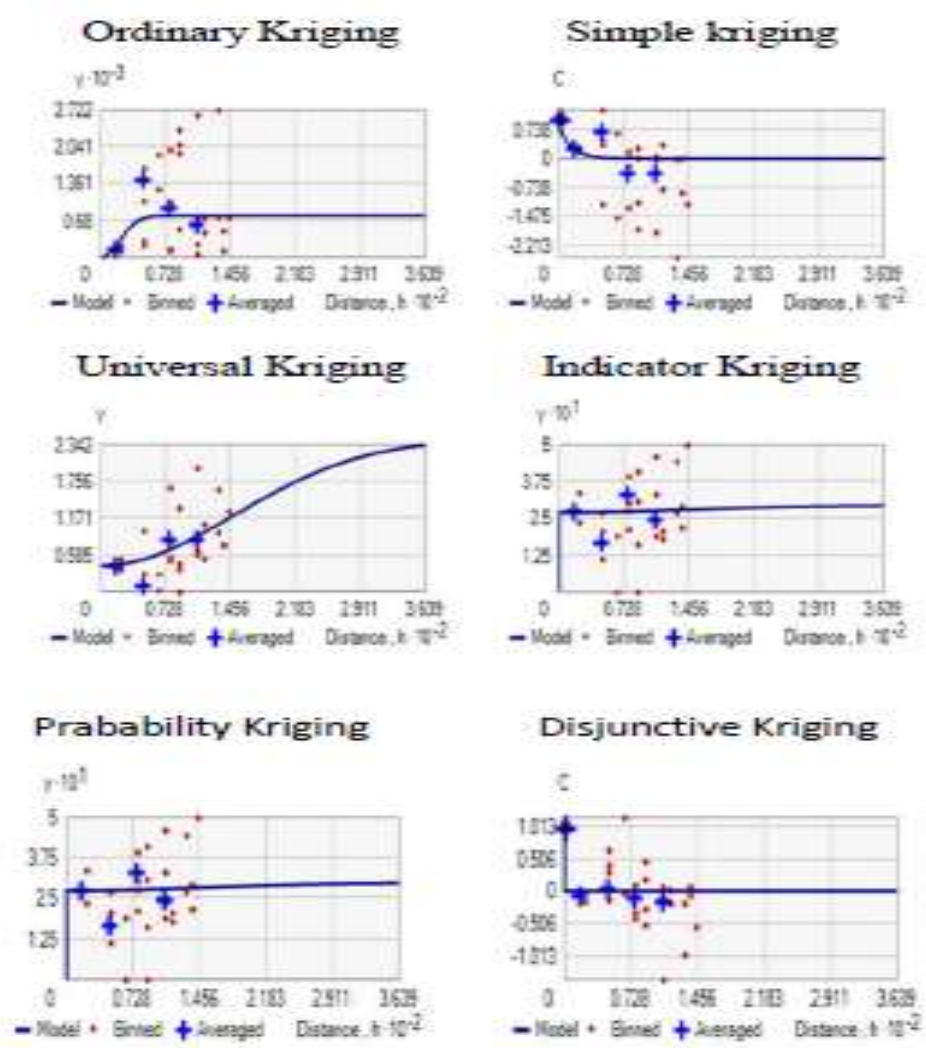

Figure 6. Semivariogram -Kriging Types

As the gap between samples boom, we anticipate the semivariance to also growth (again, due to the fact close to samples are extra related than distant samples). For kriging evaluation, the semivariogram model (the blue line in Figure 5) is used to obtain estimates for the weighting parameters.

By simulating the field values in ArcGIS [software], and getting some measurement values like RMSE values, mean, regression coefficient values and smoothness factors. Among them, we consider the RMSE measurement.

\section{Table 1. Root Mean Square Error Measurement}

$\begin{array}{cc}\text { Interpolation Techniques } & \text { RMSE[dbm] } \\ \text { Inverse Distance Weighting } & 22.44892 \\ \text { Spline/radial basis function interpolation } & 0.89566 \\ \text { Local Interpolation } & 13.09873 \\ \text { Global Interpolation } & 13.13151 \\ \text { Ordinary Kriging } & 0.841122 \\ \text { Simple kriging } & 0.7750233 \\ \text { Universal Kriging } & 0.841122 \\ \text { Indicator Kriging } & 0.521977 \\ \text { Probability kriging } & 0.536237 \\ \text { Disjunctive Kriging } & 0.841122\end{array}$




\section{Conclusion}

In this paper, it studies the better interpolation method for constructing Radio Environmental Map [REM]. Electing a suitable type of interpolation method depends on many factors. There is no universal method that is suitable for all problems. There are dissimilar methods of interpolation. Rather than assume one interpolation method is better to attempt diverse interpolation methods and compare the results to determine the best interpolation method for a given paper.

Each spatial interpolation algorithm performed differently. Although quantitative assessment is generally and extensively used in most spatial interpolation studies, it is crucial to understand that evaluation of a spatial interpolation should not rely on quantitative assessment alone. The performance of each of these algorithms was estimated based on the Root Mean Square error (RMSE) and the interpolated surface formed. From these results, it can be understood that Indicator kriging method has less RMSE of value $0.521977 \mathrm{dBm}$, provides smooth and more realistic interpolated surface and kriging methods have better accuracy [the least error]. So based on the RMSE and the interpolated surface produced, it can be concluded that Kriging methods are the best suitable methods to construct the REM.

\section{References}

[1] S. Haykin, "Cognitive radio: brain-empowered wireless communications", IEEE Journal on Selected Areas in Communications, vol. 23, no. 2, (2005), pp. 201-220.

[2] J. Mitola and G. Maguire, "Cognitive radio: Making the software radios more personal", IEEE Pers. Commun., vol. 6, (1999) August, pp. 13-18.

[3] F. Akyildiz, W. Y. Lee, M. C. Vuran and S. Mohanty, "Next generation/dynamic spectrum access/cognitive radio wireless networks: A survey, Computer Networks", vol. 50, no. 13, (2006), pp. 2127-2159.

[4] R. Zhang, Y.-C. Liang and S. Cui, "Dynamic Resource Allocation in Cognitive Radio Networks", IEEE Signal Processing Magazine, (2010), pp. 102-114.

[5] T. Yucek and H. Arslan, "A survey of spectrum sensing algorithms for cognitive radio applications", IEEE Communications Surveys and Tutorials, vol. 11, no. 1, (2009), pp. 116-130.

[6] S. Haykin, D. J. Thomson and J. H. Reed, "Spectrum sensing for cognitive radio", Proceedings of the IEEE, vol. 97, no. 5, (2009), pp. 849-877.

[7] P. Venkat Ramana and S. Narayana Reddy, "Cooperative Spectrum Sensing in Cognitive Radio Networks and Minimization of Error Probability using Optimal Decision Voting Rule", i-manager Journal on Digital Signal Processing, ISSN: 2321- 7480, vol. 2, no. 3, (2014) September, pp. 31-36.

[8] P. Venkat Ramana and S. Narayana Reddy, "Efficient Cyclostationary Detection Based Spectrum Sensing in Cognitive Radio Networks", International Journal of Engineering Trends and Technology, ISSN: 2231 5381, vol. 19, no.4, (2015) January, pp. 195-200.

[9] P. Venkat Ramana and S. Narayana Reddy, "Cyclostationary Detection Based Spectrum Sensing in Cognitive Radio Networks", International Journal of Scientific \& Engineering Research, ISSN: 22295518, Indexing-Thomson Reuters, vol. 6, no. 1, (2015) January, pp. 1005-1010.

[10] P. Venkat Ramana and S. Narayana Reddy, "Cooperative Spectrum Sensing using Energy Detection in Cognitive Radio Networks", All India Seminar on Telecommunication Switching Systems and Networks, The Institute of Engineers(India), Visakhapatnam, (2014) January, pp. 152-156.

[11] M. P. V. K. J. Riihijarvi and P. Mahonen, "Enhancing cognitive radios with spatial statistics: From radio environment maps to topology engine", 4th International Conference on Cognitive Radio Oriented Wireless Networks and Communications (CROWNCOM), (2009).

[12] M. Angjelicinoski, V. Atanasovski and L. Gavrilovska, "Comparative analysis of spatial interpolation methods for creating radio environment maps", Proc. of 19th Telecommunications Forum (TELFOR), (2011) November.

[13] P. Goovaerts, "Geo-statistics for Natural Resources Evaluation”, (Applied Geo-statistics), OUP USA, (1997).

[14] N. Cressie, "Statistics for Spatial Data", Wiley-Blackwell, (2015).

[15] J. Li and A. D. Heap, "A Review of Spatial Interpolation Methods for Environ-mental Scientists", Geoscience Australia, Record 2008/23, (2008).

[16] ArcGIS9: Using ArcGIS Geo-Statistical Analyst,"[Online]. Available: http://dusk2:geo:orst:edu/gis/geostatanalyst: pdf, 2003, accessed 29 December 2015.

[17] M. Umer, L. Kulik and E. Tanin, "Spatial interpolation in wireless sensor networks: localized algorithms for variogram modeling and kriging", Geoinfor-matica, vol. 14, (2010), pp. 101-134. 
[18] Notebook for spatial data analysis part II. Continuous spatial data analysis,"[Online]. Available: http://www:academia:edu/12744789/ accessed 20 January 2016.

[19] P. A. Burrough and R. A. McDonnell, "Principles of Geographical Information Systems" (Spatial Information Systems), OUP Oxford; 2nd edition, (1998).

[20] S. M. Kay, "Fundamentals of statistical signal processing", [Volume I], Estimation theory, Prentice Hall signal processing series. Prentice Hall, Upper Saddle River (N.J.), (1993).

\section{Authors}

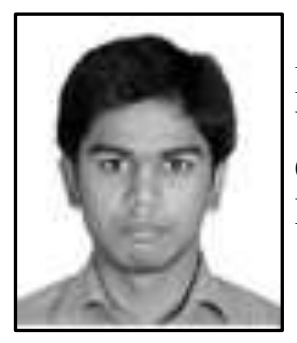

Bhaskar Reddy Konepalli, studied M.Tech in stream of "Digital Electronics and Communication Systems" during 2015-2017 in Sree Vidyanikethan Engineering College, Tirupati. Research Interests are Cognitive Radio Networks, Wireless Communication Networks, Information Theory and Coding Systems, Digital Signal Processing.

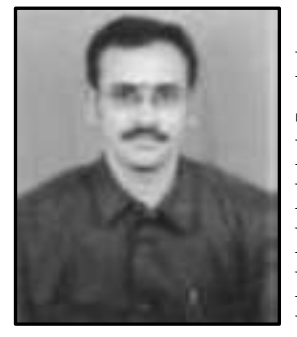

Dr. P. Venkata Ramana, he is currently Professor in the Department of Electronics and Communication Engineering at Sree Vidyanikethan Engineering College, Tirupati and Completed Ph.D. in the Department of Electronics and Communication Engineering, Sri Venkateswara University College of Engineering, Tirupati. Research Interests are Cognitive Radio Networks, Wireless Communication Networks, and Digital Signal Processing. 
International Journal of Future Generation Communication and Networking Vol. 11, No. 3 (2018) 